\title{
An Overview of Harmonic Sources in Power System
}

\author{
Imtiaz Ahmed ${ }^{1}$, Mir Zayed Shames ${ }^{1}$, Md. Muksudul Alam ${ }^{1}$ \\ ${ }^{I}$ (Department of Electrical and Electronic Engineering, Ahsanullah University of Science and Technology, \\ Dhaka, Bangladesh)
}

\begin{abstract}
Harmonics are multiples of the fundamental frequency of power system. These harmonics are creating distorted voltages and currents in the power system. Distortion of voltages and currents can affect the power quality. This article covers Harmonics sources in power system. Different Sources of harmonics in power system have been discussed.
\end{abstract}

Keywords: Current, Distortion, Frequency, Harmonics, Power System, Source, Voltage

\section{INTRODUCTION}

Harmonic waveform distortion is just one of many different disturbances that perturb the operation of electrical systems. It is also a unique problem in light of an increasing use of power electronics that basically operate through electronic switching. Different nonlinear loads produce different but identifiable harmonic spectra. This makes the task of pinpointing possible culprits of harmonic distortion more tangible. Utilities and users of electric power have to become familiar with the signatures of different waveform distortions produced by specific harmonic sources. This will facilitate the establishment of better methods to confine and remove them at the sites where they are produced. In doing this, their penetration in the electrical system affecting adjacent installations will be reduced [1], [2].

The presence of non-linear loads and the increasing number of distributed generation power systems (DGPS) in electrical grids contribute to change the characteristics of voltage and current waveforms in power systems, which differ from pure sinusoidal constant amplitude signals. Under these conditions advanced signal processing techniques are required for accurate measurement of electrical power quantities. The impact of nonlinear loads in electrical power systems has been increasing during the last decades. Such electrical loads, which introduce non-sinusoidal current consumption patterns (current harmonics), can be found in rectification frontends in motor drives, electronic ballasts for discharge lamps, personal computers or electrical appliances. Harmonics in power systems mean the existence of signals, superimposed on the fundamental signal, whose frequencies are integer numbers of the fundamental frequency. The electric utility companies should supply their customers with a supply having a constant frequency equal to the fundamental frequency, $50 / 60 \mathrm{~Hz}$, and having a constant magnitude. The presence of harmonics in the voltage or current waveform leads to a distorted signal for voltage or current, and the signal becomes non-sinusoidal signal which it should not be [3].

\section{HARMONIC SOURCES}

The main source of the harmonics is any non-linear loads that produce the voltage harmonics and current harmonics. This occurs because the resistance of the device is not a constant. The resistance in fact, changes during each sine wave. So, non linear device is one in which the current is not proportional to the applied voltage.

\subsection{Transformers}

A transformer can incur in core saturation conditions in either of the following cases: When operating above rated power when operating above rated voltage the first situation can arise during peak demand periods, and the second case can occur during light load conditions, especially if utility capacitor banks are not disconnected accordingly and the feeder voltage rises above nominal values. Distorted current under transformer saturation conditions is shown in fig 1. From the fig, it is evident that a transformer operating on the saturation region will show a nonlinear magnetizing current, which contains a variety of odd harmonics, with the third dominant. The effect will become more evident with increasing loading. No hysteresis losses are produced in an ideal lossless core. The magnetic flux and the current needed to produce them are related through the magnetizing current of the steel sheet material used in the core construction. Even under this condition, if we plot the magnetizing current vs. time for every flux value considered, the resultant current waveform would be far from sinusoidal. Sinusoidal magnetizing current is not symmetrical with respect to its maximum value because of hysteresis effect is considered. The distortion is typically due to triplen harmonics (odd multiples of three, namely, the 3rd, 9th, 15th, etc.), but mainly due to the third harmonic. This spectral component can be confined within the transformer using delta transformer connections. This will help maintain a supply voltage 
with a reasonable sinusoidal waveform [4].

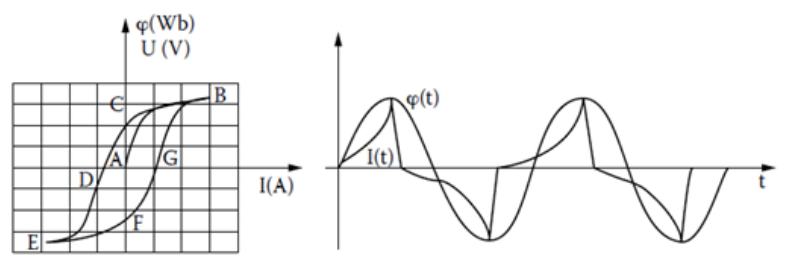

Fig. 1 Distorted current under transformer saturation conditions [1]

\subsection{Rotating Machines}

Due to rotor slots or slight irregularities in the winding patterns of a three-phase winding of a rotating machine or small asymmetries on the machine, harmonic currents can develop. These harmonics induce an electromotive force (emf) on the stator windings at a frequency equal to the ratio of speed/wavelength. The resultant distribution of magneto motive forces ( $\mathrm{mmf}$ ) in the machine produces harmonics, which is the function of speed. Additional harmonic currents can be created upon magnetic core saturation. However, these harmonic currents are usually smaller than those developed when the machines are fed through variable frequency drives (VFDs).

\subsection{Power Converters}

The increasing use of the parameters like voltage and frequency are varied to adapt to specific industrial and commercial processes has made power converters the most widespread source of harmonics in distribution systems. Electronic switching helps the task to rectify $50 / 60 \mathrm{~Hz} \mathrm{AC}$ into DC power. In DC applications, the voltage is varied through adjusting the firing angle of the electronic switching device. Basically, in the rectifying process, current is allowed to pass through semiconductor devices during only a fraction of the fundamental frequency cycle, for which power converters are often regarded as energy-saving devices. If energy is to be used as AC but at a different frequency, the DC output from the converter is passed through an electronic switching inverter that brings the DC power back to AC.

Converters can be grouped into the following categories:

- Large Power Converters

- Medium-Size Power Converters

- Low-Power Converters

- Variable Frequency Drives

\subsubsection{Large Power Converters}

Large Power Converters is a converter which large blocks of energy are transformed from AC to DC. These are used in electric utility applications. Their nominal power is in the mega-volt-ampere range. Generally, they present a much higher inductance on the $\mathrm{DC}$ than on the $\mathrm{AC}$ side. Therefore, the $\mathrm{DC}$ current is practically constant and the converter acts as a harmonic voltage source on the DC side and as a harmonic current source on the AC side. Furthermore, all resultant currents are the same in all phases in a perfectly balanced system.

\subsubsection{Medium-Size Power Converters}

Medium-Size Power Converters' range is Kilo-volt-ampere size. These are found in increasing numbers in industry. The first applications in the industry were for DC motor speed control that still represents the major market for these types of converters. However, they are increasingly used in AC induction motor speed control. Similar to large-size power converters, the fifth harmonic can reach amplitudes that range from one fifth to one third the fundamental rated current.

\subsubsection{Low-Power Converters}

UPS (Uninterruptible Power Supply) units, discharge lamps, television sets, computers, fax machines are among these low kilo-volt-ampere- size power converter applications. If they are additionally provided with UPSs to handle voltage sags and power supply interruptions, the amounts of harmonic currents can substantially increase [5]. 


\subsubsection{Variable Frequency Drives}

Variable frequency drive (VFD) usage has increased dramatically in HVAC applications. The VFDs are now commonly applied to air handlers, pumps, chillers and tower fans. A better understanding of VFDs will lead to improved application and selection of both equipment and HVAC systems.

VFDs draw current from the line only when the line voltage is greater than the DC Bus voltage inside the drive. This occurs only near the peaks of the sine wave. As a result, all of the current is drawn in short intervals (i.e., at higher frequencies). Variation in VFD design affects the harmonics produced [6].

\subsection{Fluorescent Lamps}

For pure and stable sinusoidal voltages, electric power systems are designed. Due to the incessantly growing demand for electricity, the levels of harmonic distortions of voltage and current waveforms in power systems have steadily increased. Harmonic distortions affect sensitive equipment connected to the power networks and are especially problematic for compact fluorescent lamps.

Fluorescent Lamps are highly nonlinear in their operation. It gives rise to odd harmonic currents of important magnitude. In fluorescent lamp, magnetic core inductors or chokes contained inside the start ballasts function to limit the current to the tube. Likewise, they use a capacitor to increase the efficiency of the ballast by increasing its power factor. Electronic ballasts operate at higher frequency, which permits the use of smaller reactors and capacitors. The use of higher frequencies allows them to create more light for the same power input. This is used to reduce the input power [1].

\subsection{Arcing Devices}

The voltage-current characteristics of electric arcs are highly non-linear. Following arc ignition the voltage decreases due to the short-circuit current, the value of which is only limited by the power system impedance. The main harmonic sources in this category are the electric arc furnace. It discharges type lighting with magnetic ballasts.

The harmonics produced by electric arc furnaces are not definitely predicted due to variation of the arc feed material. The arc current is highly nonlinear and reveals a continuous spectrum of harmonic frequencies of both integer and non integer order. Due to the physical Phenomenon of the melting with a moving electrode and molten material, the arc furnace load gives the worst distortion. The arc current wave may not be same from cycle to cycle.

\section{FUTURE SOURCES OF HARMONICS}

The sources of harmonics in the electrical system of the future will be various and numerous. Due to increasing use of sensitive electronics in personal computers, multimedia, digital communications and industrial automated processes, the problem grows complicated.

Generally, which are not regarded as large generators of harmonics, may be lining up to join current harmonic producers. Photovoltaic, wind, natural gas, carbonate full cells and even hydrogen are expected to play increasingly important roles in managing the electricity needs of the future.

\section{CONCLUSION}

This paper discussed different sources of harmonic in power system. Transformers, rotating machines, power converters, fluorescent lamps and Arcing Devices are the main source of harmonic in power system. Harmonic current is produced by harmonic. Harmonic currents can cause overload in wiring and transformers, can produce heat, and in extreme cases, fire.

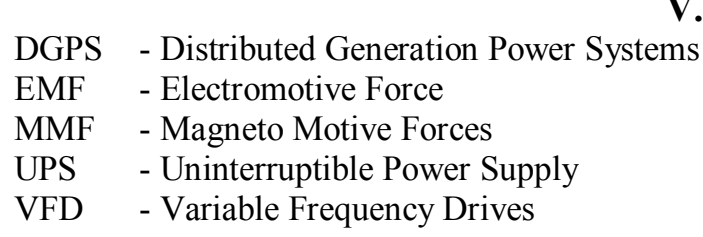

\section{ACRONYMS}

\section{REFERENCES}

[1] C. Francisco and R. DeLa, "Harmonics and Power Systems", CRC Press Taylor \& Francis Group, LLC, 2006

[2] K. D. Patil and W. Z. Gandhare, "Effects of Harmonics in Distribution Systems on Temperature Rise and Life of XLPE Power Cables", Power and Energy Systems (ICPS), pp. 1 - 6, Chennai, 22-24 December 2011

[3] J. Arrillaga, D. A. Bradley and P.S. Bodger, "Power System Harmonics", John Wiley \& Sons, New York, 1985

[4] R. D. Henderson and P. J. Rose, "Harmonics: The Effects on Power Quality and Transformers", Industry Applications, IEEE Transactions, Vol. 30 , Issue: 3, pp. 528 - 532 , May/June 1994

[5] M. Rashid, "Power Electronics: Circuits, Devices, and Applications", 3rd Edition, Prentice Hall New Jersey, USA, 2004

[6] Variable Frequency Drive by Carrier Corporation Syracuse, New York, October 2005 